\title{
Kidney Extraskeletal Osteosarcoma
}

National Cancer Institute

\section{Source}

National Cancer Institute. Kidney Extraskeletal Osteosarcoma. NCI Thesaurus. Code C6181.

An osteosarcoma arising from the kidney. 